\title{
Design and Baseline Characteristics of the Finerenone in Reducing Cardiovascular Mortality and Morbidity in Diabetic Kidney Disease Trial
}

\author{
Luis M. Ruilope $^{a-c}$ Rajiv Agarwal ${ }^{d}$ Stefan D. Ankere George L. Bakris ${ }^{f}$ \\ Gerasimos Filippatos $^{9} \quad$ Christina Nowack $^{\mathrm{h}}$ Peter Kolkhof ${ }^{\mathrm{i}}$ Amer Joseph $^{\mathrm{j}}$ \\ Nicole Mentenichk ${ }^{k}$ Bertram Pitt ${ }^{\prime}$ FIGARO-DKD study investigators
}

${ }^{a}$ Cardiorenal Translational Laboratory and Hypertension Unit, Institute of Research imas12, Madrid, Spain; ${ }^{b} \mathrm{CIBER}-$ CV, Hospital Universitario 12 de Octubre, Madrid, Spain; ${ }^{C}$ Faculty of Sport Sciences, European University of Madrid,

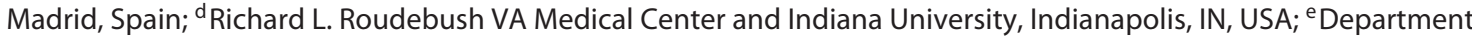
of Cardiologsupply, and Berlin Institute of Health Center for Regenerative Therapies, German Centre for Cardiovascular Research Partner Site Berlin, Charité Universitätsmedizin, Berlin, Germany; ${ }^{f}$ Department of Medicine, University of Chicago, Chicago, IL, USA; ${ }^{9}$ Department of Cardiology, Attikon University Hospital, Athens, Greece, and University of Cyprus, Medical School, Nicosia, Cyprus; ${ }^{\mathrm{h}}$ Research and Development, Clinical Development

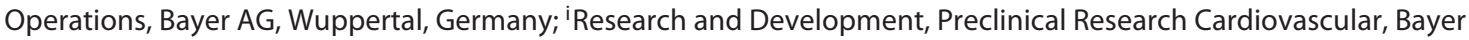
AG, Wuppertal, Germany; ${ }^{j}$ Research and Development, Clinical Development, Bayer AG, Berlin, Germany; ${ }^{\mathrm{k}}$ Research and Development, Statistics and Data Insights, Bayer AG, Wuppertal, Germany; 'Department of Medicine, University of Michigan School of Medicine, Ann Arbor, MI, USA

\section{Keywords}

Kidney · Mineralocorticoid · Diabetes · Aldosterone ·

Outcomes $\cdot$ Clinical

\begin{abstract}
Background: Among people with diabetes, those with kidney disease have exceptionally high rates of cardiovascular (CV) morbidity and mortality and progression of their underlying kidney disease. Finerenone is a novel, nonsteroidal, selective mineralocorticoid receptor antagonist that has shown to reduce albuminuria in type 2 diabetes (T2D) patients with chronic kidney disease (CKD) while revealing only a low risk of hyperkalemia. However, the effect of finerenone on CV and renal outcomes has not yet been investigated in longterm trials. Patients and Methods: The Finerenone in Reducing CV Mortality and Morbidity in Diabetic Kidney Disease (FIGARO-DKD) trial aims to assess the efficacy and safety of finerenone compared to placebo at reducing clinically im-
\end{abstract}

\begin{tabular}{ll}
\hline KARGER & $\begin{array}{l}\text { (c) } 2019 \text { The Author(s) } \\
\text { Published by S. Karger AG, Basel }\end{array}$ \\
E-Mail karger@karger.com & $\begin{array}{l}\text { This article is licensed under the Creative Commons Attribution- } \\
\text { NonCommercial-NoDerivatives 4.0 International License (CC BY- } \\
\text { NC-ND) (http://www.karger.com/Services/OpenAccessLicense). } \\
\text { Usage and distribution for commercial purposes as well as any dis- } \\
\text { tribution of modified material requires written permission. }\end{array}$
\end{tabular}

portant CV and renal outcomes in T2D patients with CKD. FIGARO-DKD is a randomized, double-blind, placebo-controlled, parallel-group, event-driven trial running in 47 countries with an expected duration of approximately 6 years. FIGARO-DKD randomized 7,437 patients with an estimated glomerular filtration rate $\geq 25 \mathrm{~mL} / \mathrm{min} / 1.73 \mathrm{~m}^{2}$ and albuminuria (urinary albumin-to-creatinine ratio $\geq 30$ to $\leq 5,000 \mathrm{mg} / \mathrm{g}$ ). The study has at least $90 \%$ power to detect a $20 \%$ reduction in the risk of the primary outcome (overall two-sided significance level $a=0.05$ ), the composite of time to first occurrence of CV death, nonfatal myocardial infarction, nonfatal stroke, or hospitalization for heart failure. Conclusions: FIGARO-DKD will determine whether an optimally treated cohort of T2D patients with CKD at high risk of CV and renal events will experience cardiorenal benefits with the addition of fi-

FIGARO-DKD Phase 3 investigators and site list are included in the online supplementary files
Luis M. Ruilope, MD

Centro de Investigación, Instituto de Investigación imas 12

Hospital Universitario 12 de Octubre

Avenida de Córdoba s/n, ES-28041 Madrid (Spain)

E-Mail ruilope@icloud.com 
nerenone to their treatment regimen. Trial Registration: EudraCT number: 2015-000950-39; ClinicalTrials.gov identifier: NCT02545049.

(c) 2019 The Author(s)

Published by S. Karger AG, Basel

\section{Background}

Although rates of diabetes-related complications have declined substantially in the past 2 decades, the large burden of disease persists because of the continued increase in the prevalence of diabetes [1]. The number of people with diabetes will rapidly increase, and it is estimated that by 2040642 million people aged $20-79$ years will have diabetes [2].

High blood glucose, blood pressure, serum cholester$\mathrm{ol}$, and body mass index are risk factors for cardiovascular disease (CVD), and some of these factors also increase the risk of chronic kidney disease (CKD) and type 2 diabetes (T2D) [3].

Death related to cardiovascular $(\mathrm{CV})$ causes is the main competing outcome to development of end-stage renal disease (ESRD) in patients with CKD. For patients with CKD stage 3 (estimated glomerular filtration rate [eGFR] $\leq 60 \mathrm{~mL} / \mathrm{min} / 1.73 \mathrm{~m}^{2}$ ), the risk of death, mainly due to CV events, is over 10 times higher than the risk of progressing to ESRD [4]. People with diabetes have a 10 -year cumulative all-cause mortality of $11.5 \%$, which is $3.9 \%$ higher than the reference group without diabetes or CKD (7.7\% cumulative 10-year mortality). In patients with CKD and diabetes, however, standardized mortality was $31.1 \%$, representing an absolute risk difference from the reference group of $23.4 \%$, even after adjustment for demographics and CV risk factors [5].

A three-fold increase in all-cause mortality and a 16-year loss in life expectancy were found in a recent investigation in patients with diabetes with early kidney disease. Mortality was nearly twice as high as for early kidney disease alone or diabetes without kidney disease, and lifespan loss was significantly higher than for any of the single conditions ( 6 years of life span loss for early kidney disease and 10 years for people with diabetes) [6].

It can be concluded that the excess mortality among people with diabetes appears to be largely limited to the subgroup with kidney disease and can be explained by their high burden of CVDs [5, 7].

Considering the high mortality and morbidity in patients with $\mathrm{CKD}$, primary prevention of its development but also efforts to hinder its progression once established are of utmost importance.
According to guidelines, CV risk factors should be systematically assessed at least annually for prevention and management of CVD in people with diabetes, and risk factors such as blood pressure, blood glucose, or lipid levels controlled by applying lifestyle intervention and pharmacological strategies. In addition, antiplatelet therapy may be considered as primary prevention in high-risk patients but is clearly recommended in patients with diabetes with established CVD [8].

Additionally, recently approved glucose-lowering therapies have demonstrated reductions in $\mathrm{CV}$ event rates. In a recent meta-analysis of all $\mathrm{CV}$ outcome trials in patients with $\mathrm{T} 2 \mathrm{D}$, sodium-glucose cotransporter-2 (SGLT2) inhibitors reduced major CV events (i.e., myocardial infarction, stroke, or CV death) by $11 \%$, with benefit only seen in patients with atherosclerotic disease and not in those without [9]. SGLT2 inhibitors reduced the risk of CV death or hospitalization for heart failure by $23 \%$, with a similar benefit in patients with and without atherosclerotic CVD and with and without a history of heart failure. From mid-June 2019 onwards, among patients with T2D and albuminuria $>300 \mathrm{mg} / \mathrm{g}$, SGLT2 inhibitors are recommended if eGFR is $>30 \mathrm{~mL} / \mathrm{min} / 1.73 \mathrm{~m}^{2}$ to protect from both $\mathrm{CV}$ events and renal disease progression [8].

While consequent control of risk factors, optimization of therapy, and changes in lifestyle have led to significant reductions in CV events over the last years, event rates in patients with CKD (stage 3 and lower) are still high [9]. In particular, in T2D patients with CKD but without CVD in the medical history, new treatments such as SGLT2 inhibitors showed only moderate benefits on major CV events [9] or were not sufficiently tested in CV outcomes trials with glucagon-like peptide 1 (GLP-1) analogs such as liraglutide or semaglutide [1012].

Plasma aldosterone concentrations and mineralocorticoid receptor (MR) overactivation are associated with an enhanced risk of CV injury by exerting deleterious effects on the heart, kidney, brain, and peripheral vasculature in preclinical investigations [13-16]. MR antagonists (MRAs) are evidence-based therapy in symptomatic patients with heart failure and reduced ejection fraction (HFrEF) [17]. Subgroup analyses of previous studies with the 2 steroidal MRAs spironolactone and eplerenone showed that MR antagonism decreases the risk of $\mathrm{CV}$ events and sudden death in patients with HFrEF and impaired kidney function [18-22]. Because of this evidence, similar morbidity and mortality benefits might also accrue in T2D patients with CKD [23]. 
Spironolactone is not only a direct MRA but also a pharmacologically active prodrug generating active metabolites, such as 7 alpha-thiomethyl-spironolactone and canrenone, which have long half-lives and contribute to important side effects such as gynecomastia and hyperkalemia. Depending on the dose, up to $52 \%$ of male patients treated with spironolactone develop gynecomastia due to its nonspecific interaction with the homologous androgen receptor, further limiting the use of this drug $[24,25]$. Eplerenone represents the second generation of steroidal MRAs that does not cause gynecomastia and should be used with precaution in those with T2D and microalbuminuria because of the risk of developing hyperkalemia [26]. Therefore, the widespread clinical use of MRAs is limited by the fear and risk of developing hyperkalemia [27], and, if initiated, MRAs are often discontinued within the first several months due to hyperkalemia [28]. For this reason, there is a lack of data on renal and CV outcomes on available steroidal MRAs, eplerenone, and spironolactone, in T2D patients with CKD [29].

Finerenone is a novel nonsteroidal, selective MRA that has greater MR selectivity than spironolactone and better MR affinity than eplerenone in vitro [30, 31]. Furthermore, unlike spironolactone and eplerenone, which reach higher concentrations in renal tissue in comparison to cardiac tissue [32], finerenone is distributed relatively equally between the heart and the kidneys, at least in rodents [33]. Last, its nonsteroidal structure allows it to bind to the MR with high affinity and to inhibit recruitment of transcriptional coactivators involved in the expression of hypertrophic and profibrotic genes more effectively in comparison to the steroidal MRAs [34, 35].

In a trial involving T2D patients with the clinical diagnosis of CKD (MRA Tolerability Study-Diabetic Nephropathy), finerenone compared to placebo showed only small mean changes in serum potassium and a low incidence of hyperkalemia, leading to a low number of discontinuations with no evidence of a dose-response relationship. Furthermore, finerenone compared to placebo demonstrated a statistically significant reduction in urinary albumin-to-creatinine ratio (UACR) at doses starting from $7.5 \mathrm{mg}$ onwards on top of standard of care, including an angiotensin-converting enzyme inhibitor (ACEI) or angiotensin receptor blocker (ARB) after 90 days of treatment [36].

These findings provided the rationale for initiating a large-scale phase III program with finerenone investigating $\mathrm{CV}$ and renal outcomes in T2D patients with all stages of CKD in 2 large-scale outcome trials. In this paper, we describe the design of Finerenone in Reducing CV Mortality and Morbidity in Diabetic Kidney Disease (FIGARO-DKD; ClinicalTrials.gov identifier NCT02545049) and present the baseline characteristics of patients enrolled into this trial. The design and baseline characteristics of the Finerenone in Reducing Kidney Failure and Disease Progression in diabetic kidney disease (FIDELIO-DKD, ClinicalTrials.gov identifier: NCT02540993) renal outcome trial are also in this issue of Am J Nephrol.

\section{Patients and Methods}

\section{General Description}

FIGARO-DKD is an international, multicenter, randomized, double-blind, placebo-controlled, parallel-group, event-driven study formally designed to assess whether finerenone reduces cardiorenal morbidity and mortality in T2D patients with CKD when used in addition to standard of care.

\section{Study Population}

The inclusion and exclusion criteria are summarized in Table 1. Patients included in the trial were aged $\geq 18$ years with T2D and the clinical diagnosis of CKD, pretreated with ACEIs or ARBs for at least 4 weeks prior to the run-in visit, and had serum potassium $\leq 4.8 \mathrm{mEq} / \mathrm{L}$ at both the run-in and screening visits. Patients had to have persistent high albuminuria (defined as UACR $\geq 30 \mathrm{mg} / \mathrm{g}$ but $<300 \mathrm{mg} / \mathrm{g}$ ) and eGFR $\geq 25$ but $\leq 90 \mathrm{~mL} / \mathrm{min} / 1.73 \mathrm{~m}^{2}$, or persistent very high albuminuria (defined as UACR $\geq 300 \mathrm{mg} / \mathrm{g}$ ) and an $\mathrm{eGFR} \geq 60 \mathrm{~mL} / \mathrm{min} / 1.73 \mathrm{~m}^{2}$.

Two recruitment caps, both affecting patients with high albuminuria at screening, were prespecified and closed per region. The first cap limited the randomization of patients with eGFR $\geq 60 \mathrm{~mL} /$ $\mathrm{min} / 1.73 \mathrm{~m}^{2}$ in the medical history to approximately $10 \%$ of all patients randomized with high albuminuria at screening, and the second cap limited the randomization of patients without a history of CVD to approximately $40 \%$ of all patients randomized with high albuminuria at screening.

\section{Study Design}

The study design of FIGARO-DKD is illustrated in Figure 1. After signing the informed consent form, a run-in visit was performed. If the patient met all inclusion criteria and none of the exclusion criteria, a mandatory run-in period that could last between a minimum of 4 and a maximum of 16 weeks started. At the end of the run-in period, a screening visit to confirm the patient's eligibility again took place with subsequent randomization within 2 weeks.

To account for the high variability of UACR and eGFR, patients were permitted to switch after either the run-in or the screening visit between FIGARO-DKD and the second study of the program, FIDELIO-DKD (ClinicalTrials.gov identifier: NCT02540993), before being randomized. As CKD in T2D is a disease continuum and variations in eGFR and UACR within the boundaries of this disease continuum do not affect the clinical risk, the switching of patients prior to randomization was deemed justified and neces- 
Table 1. Inclusion and exclusion criteria

\begin{tabular}{|c|c|}
\hline \multirow[t]{7}{*}{ Inclusion criteria } & Written, informed consent signed before any study-specific procedure \\
\hline & Men or women aged 18 years and older \\
\hline & Women of childbearing potential with a negative pregnancy test and agreeing to use adequate contraception \\
\hline & Patients with type 2 diabetes mellitus as defined by the American Diabetes Association \\
\hline & $\begin{array}{l}\text { Patients with a clinical diagnosis of DKD based on either of the following criteria at the run-in and screening visits: } \\
\text { - Persistent high albuminuria defined as UACR } \geq 30 \mathrm{mg} / \mathrm{g}(\geq 3.4 \mathrm{mg} / \mathrm{mmol}) \text { but }<300 \mathrm{mg} / \mathrm{g}(<33.9 \mathrm{mg} / \mathrm{mmol}) \text { and eGFR } \geq 25 \\
\text { but } \geq 90 \mathrm{~mL} / \mathrm{min} / 1.73 \mathrm{~m}^{2} \text { (CKD-EPI) in the medical history } \\
\text { or } \\
\text { - Persistent very high albuminuria defined as UACR } \geq 300 \mathrm{mg} / \mathrm{g}(\geq 33.9 \mathrm{mg} / \mathrm{mmol}) \text { and eGFR } \geq 60 \mathrm{~mL} / \mathrm{min} / 1.73 \mathrm{~m}{ }^{2} \text { (CKD-EPI) }\end{array}$ \\
\hline & Prior treatment with ACEIs and ARBs \\
\hline & Serum potassium $\leq 4.8 \mathrm{mEq} / \mathrm{L}$ at both the run-in and screening visits \\
\hline
\end{tabular}

Exclusion criteria

Medical and surgical history Known significant nondiabetic renal disease, including clinically relevant renal artery stenosis

$\mathrm{UACR}>5,000 \mathrm{mg} / \mathrm{g}(>565 \mathrm{mg} / \mathrm{mmol})$ at the run-in or screening visit

Glycosylated hemoglobin $>12 \%(>108 \mathrm{mmol} / \mathrm{mol})$ at the run-in or screening visit

Uncontrolled arterial hypertension with mean sitting SBP $\geq 170 \mathrm{~mm} \mathrm{Hg}$ or mean sitting DBP $\geq 110 \mathrm{~mm} \mathrm{Hg}$ at the run-in visit or mean sitting SBP $\geq 160 \mathrm{~mm} \mathrm{Hg}$ or mean sitting DBP $\geq 100 \mathrm{~mm} \mathrm{Hg}$ at the screening visit

SBP $<90 \mathrm{~mm} \mathrm{Hg}$ at the run-in or screening visit

Patients with a clinical diagnosis of chronic heart failure with reduced ejection fraction and persistent symptoms (New York Heart Association classes II-IV) at the run-in visit

Stroke, transient ischemic cerebral attack, acute coronary syndrome, or hospitalization for worsening heart failure, in the last 30 days prior to the screening visit

Dialysis for acute renal failure within 12 weeks prior to the run-in visit

Renal allograft in place or a scheduled kidney transplant within the next 12 months from the run-in visit

Known hypersensitivity to the study treatment (active substance or excipients)

Addison's disease

Hepatic insufficiency classified as Child-Pugh C

Medication and drug use Concomitant therapy with eplerenone, spironolactone, any renin inhibitor, or potassium-sparing diuretic which cannot be discontinued at least 4 weeks prior to the screening visit

Concomitant therapy with both ACEIs and ARBs that cannot be discontinued for the purpose of the studies

Concomitant therapy with potent cytochrome P450 isoenzyme 3A4 inhibitors or inducers (to be stopped at least 7 days before randomization)

Other

Any other condition or therapy that would make the patient unsuitable for the studies and will not allow participation for the full planned study period (e.g., active malignancy or other condition limiting life expectancy to $<12$ months)

Pregnant or breast-feeding or intention to become pregnant during the studies

Previous assignment to treatment during this studies

Previous (within 30 days prior to randomization) or concomitant participation in another clinical study (i.e., Phase I-III clinical studies) with investigational medicinal product(s), except for participation in the run-in and screening periods of Studies $17,530 / 16,244$

Close affiliation with the investigational site; for example, a close relative of the investigator, dependent person (e.g., employee or student of the investigational site)

ACEI, angiotensin-converting enzyme inhibitor; ARB, angiotensin receptor blocker; CKD-EPI, Chronic Kidney Disease Epidemiology Collaboration; DBP, diastolic blood pressure; DKD, diabetic kidney disease; eGFR, estimated glomerular filtration rate; SBP, systolic blood pressure; UACR, urinary albumin-tocreatinine ratio. 


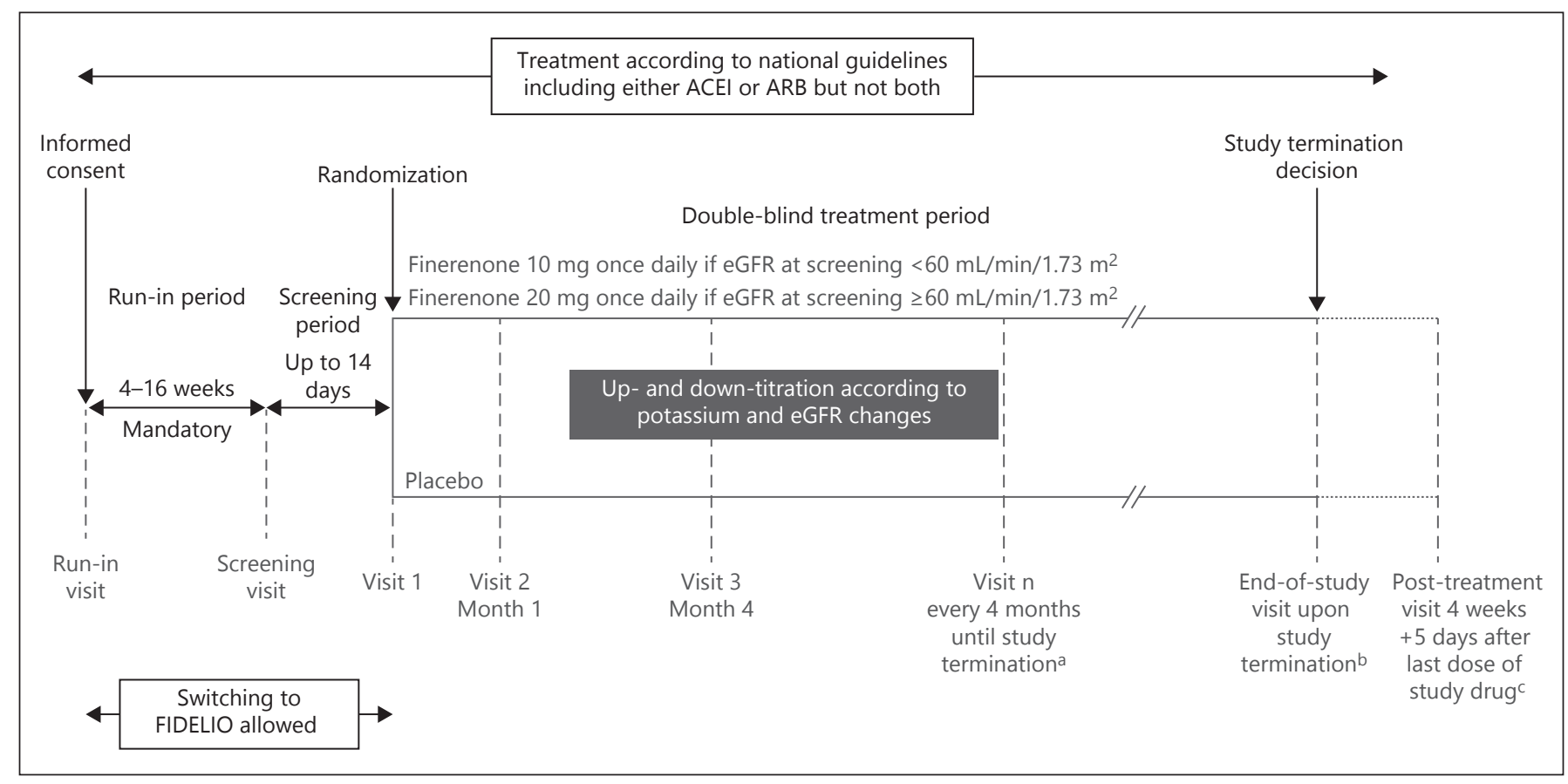

Fig. 1. Study design of FIGARO-DKD trial. ${ }^{\text {a }}$ Scheduled visits will continue even if the study drug treatment is discontinued; ${ }^{b}$ endof-study visit will take place only after the sponsor has officially terminated the study; ${ }^{\mathrm{c}}$ for all patients who have received the study

sary to reduce the burden on candidate study participants (run-in and screening assessments were not repeated after switching). A switchover from either trial was permitted only once.

Due to the anticipated recruitment period of $>2$ years and the progressive nature of the underlying disease, rescreening was permitted once. Conditions for rescreening were amended in 2017 to shorten the rescreening period from 6 to 3 months and allow rescreening even if the reason for initial screen failure was an elevated blood potassium value (serum potassium $>4.8 \mathrm{mEq} / \mathrm{L}$ ).

Eligible patients were randomized 1:1 via an interactive telephone/web-based system to receive once-daily treatment with either finerenone or placebo. Treatment assignment was determined by computer-generated random sequence with stratification by region (North America, Latin America, Europe, Asia, Other), eGFR category at screening ( 25 to $<45,45$ to $<60$, and $\geq 60 \mathrm{~mL} /$ $\mathrm{min} / 1.73 \mathrm{~m}^{2}$ ), category of albuminuria at screening (high or very high albuminuria), and history of CVD (present or absent). The definition of CVD for stratification purposes can be found in online supplementary Table 1 (for all online suppl. material, see www.karger.com/doi/10.1159/000503712).

The starting dose of study drug was selected based on the eGFR measured at the screening visit: patients started on finerenone $10 \mathrm{mg} /$ day or matching placebo if eGFR was 25 to $<60 \mathrm{~mL} /$ $\mathrm{min} / 1.73 \mathrm{~m}^{2}$; if eGFR was $\geq 60 \mathrm{~mL} / \mathrm{min} / 1.73 \mathrm{~m}^{2}$, the starting dose was $20 \mathrm{mg} /$ day. Uptitration of study drug to $20 \mathrm{mg} /$ day was permitted at any time after Visit 2 (Month 1); downtitration to $10 \mathrm{mg}$ / day at any time after start of treatment. Study participants were instructed to take the study drug tablet orally, once daily at approximately the same time every day.

Design and Baseline Characteristics of the FIGARO Trial drug. ACEI, angiotensin-converting enzyme inhibitor; ARB, angiotensin receptor blocker; eGFR, estimated glomerular filtration rate; FIDELIO, Finerenone in Reducing Kidney Failure and Disease Progression.

Central laboratory values, including serum potassium and serum creatinine, are obtained at all study visits and used for confirmation of patients' eligibility, safety monitoring, renal endpoint reporting, and end-of-study statistical analysis. From Visit 1 onwards, blood potassium and serum creatinine are measured at all on-treatment visits at a local laboratory. The investigators are instructed to use these local values for dose titrations and safety monitoring. Patients' safety is monitored also with physical examinations (including measurements of weight and vital signs) and 12-lead electrocardiograms at regular intervals throughout the study. Patients are assessed for adverse events and study endpoints at each visit and are requested to complete health-related quality-of-life questionnaires (EQ-5D-5L and Kidney Disease Quality of Life) at baseline and yearly thereafter.

The study protocol was approved by International Review Boards, independent ethics committees, and competent authorities according to national and international regulations. FIGARODKD is conducted in accordance with the ICH Harmonised Tripartite Guideline for Good Clinical Practice. All participants provided written, informed consent prior to entering the study.

\section{Objectives and Endpoints}

The primary endpoint of FIGARO-DKD is a composite of time to first occurrence of CV mortality and morbidity, as measured by the composite endpoint of time to first occurrence of CV death, nonfatal myocardial infarction, nonfatal stroke, or hospitalization for heart failure. 
Fig. 2. Statistical testing strategy of primary and secondary efficacy endpoints for FIGARO-DKD. CV, cardiovascular; UACR, urinary albumin-to-creatinine ratio.

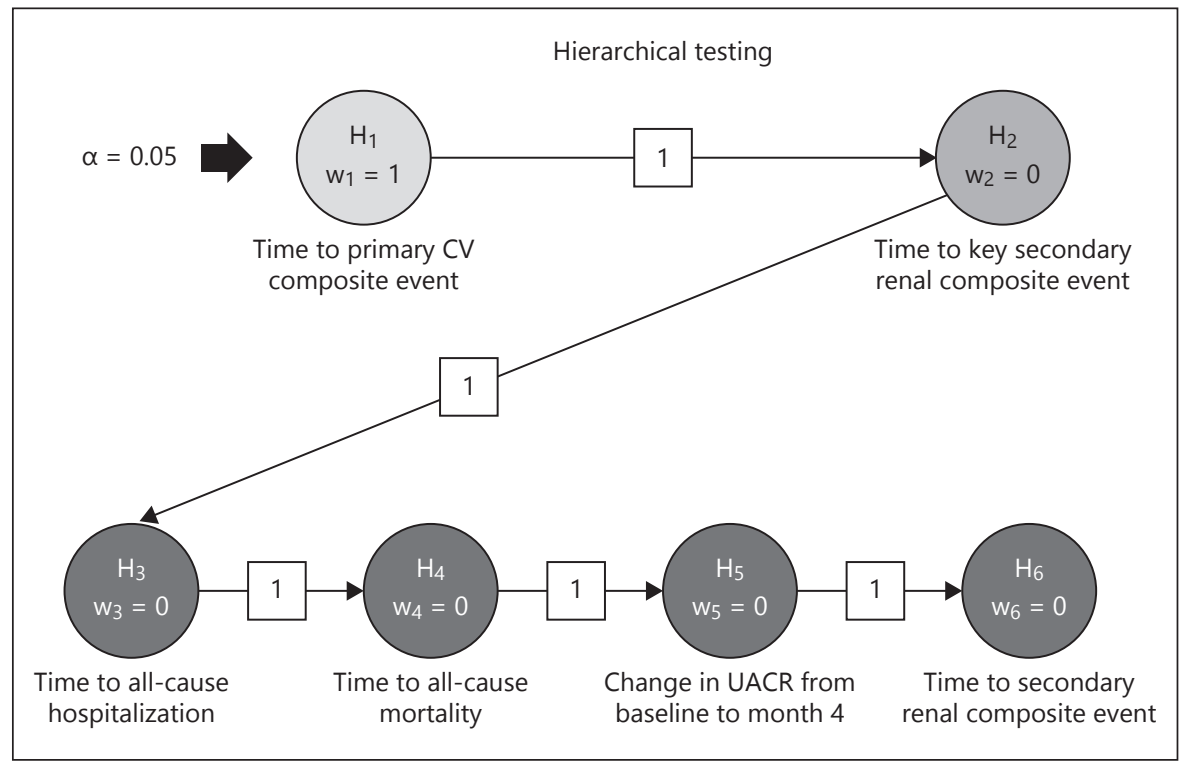

The main prespecified secondary endpoint is a composite of time to first occurrence of kidney failure, a sustained decrease of eGFR $\geq 40 \%$ from baseline over at least 4 weeks, or renal death.

The component kidney failure of this secondary endpoint is defined as the occurrence of either ESRD or eGFR $<15 \mathrm{~mL} /$ $\mathrm{min} / 1.73 \mathrm{~m}^{2}$, confirmed by a second measurement at the earliest 4 weeks after the initial measurement. ESRD is defined as the initiation of chronic dialysis (hemo- or peritoneal dialysis) for at least 90 days or renal transplantation. The eGFR threshold of $15 \mathrm{~mL} /$ $\mathrm{min} / 1.73 \mathrm{~m}^{2}$ is consistent with the definition of kidney failure from Kidney Disease: Improving Global Outcomes [37] and was chosen in order to include an objective component to the endpoint because the decision to initiate dialysis therapy or kidney transplantation may be affected by factors other than eGFR.

Other secondary endpoints include time to all-cause hospitalization, time to all-cause mortality, change in UACR from baseline to 4 months, and a composite endpoint of time to first occurrence of kidney failure or sustained decrease in eGFR $\geq 57 \%$ from baseline over at least 4 weeks or renal death.

\section{Statistical Considerations}

\section{Statistical Analyses}

The primary efficacy endpoint for the statistical analysis is the time to first occurrence of the composite endpoint of $\mathrm{CV}$ death and nonfatal CV event, the latter of which consists of myocardial infarction, stroke, or hospitalization for heart failure. Events included in the primary analysis will be counted from the day of randomization onwards until the planned end-of-study visit following the study sponsor's decision to terminate the study. Patients without a primary efficacy endpoint event will be censored at the date of their last contact up to and including the end-of-study visit or their date of non-CV death.

In order to test for superiority of finerenone over placebo in prolonging the time to the first primary efficacy endpoint event, the null hypothesis of equal hazards in the 2 treatment groups will be evaluated via a log-rank test, stratified by the stratification factors region, eGFR category at screening, category of albuminuria at screening, and history of CVD. The treatment effect will be expressed as a hazard ratio with a corresponding confidence interval from a stratified Cox regression model. The statistical analyses will follow the intention-to-treat principle and will be based primarily on the full analysis set consisting of all randomized patients considered valid for analysis. An analysis on the per protocol set, excluding patients with relevant deviations from the protocol, will be conducted as a sensitivity analysis in which only events occurring within 30 days after treatment discontinuation will be considered.

In order to control the multiplicity arising from multiple tests, a hierarchical testing procedure will be used for the primary efficacy endpoint followed by the secondary efficacy endpoints as in the order given above. Within the hierarchy of the primary and secondary efficacy endpoints, the full alpha level of $5 \%$ is forwarded to the next null hypothesis as long as the previous null hypothesis is rejected, otherwise confirmatory testing stops at this point. As described under Powering and Sample Size, the actual significance levels used at the final analysis will be slightly adjusted based on the formal interim analysis. The testing strategy is further illustrated in Figure 2. In order to declare the study successful, it will be considered sufficient to reject the null hypothesis related to the primary CV composite endpoint.

\section{Powering and Sample Size}

As an event-driven study, FIGARO-DKD has a power of $90 \%$ to detect a $20 \%$ relative risk reduction of finerenone versus placebo in the primary efficacy endpoint (equaling a hazard ratio of 0.8 ) based on a total of between 970 and 976 primary CV events and using the log-rank test at a two-sided significance level of $\alpha=5.0 \%$. Further assumptions at the planning stage included an annual placebo event rate of $8 \%$ (assumed to be unaffected by treatment discontinuations), a common annual lostto-follow-up rate of $0.7 \%$ in both treatment groups, an annual finerenone discontinuation rate of $5 \%$, and a total treatment duration between 44 and 48 months, consisting of a recruitment 
period of 33 and 41 months with an equal recruitment pattern during the accrual period and a maximum treatment period of the last recruited patient of 11 and 7 months, respectively. Taking the ramp-up time during recruitment into consideration, this leads to an estimated required number of approximately 6,400 patients to be randomized. Assuming a screening failure rate of $50 \%$, this results in 12,800 patients to be screened. To account for the lower than assumed event rates for the primary endpoint as observed during the conduct of the trial, the originally planned number of randomized patients was increased by approximately 1,000 patients.

One formal interim analysis is planned to be conducted when 651 primary efficacy endpoint events (i.e., $2 / 3$ of the finally intended number) have been observed. To guide the decision regarding early stopping of the study for success at the interim analysis, the Haybittle-Peto rule will be used, requiring a two-sided $p$ value below 0.00270 for both the null hypotheses corresponding to the primary efficacy and the key secondary renal composite endpoint to be rejected and leading to a minimal alpha adjustment for the respective tests at the final analysis stage. Additionally, for lack of efficacy, a nonbinding futility approach will be utilized to allow stopping the study at the interim analysis stage if the conditional power of rejecting the null hypothesis related to the primary endpoint at the final stage given the interim data fall to an unacceptably low level of $10 \%$ or less.

\section{Additional Statistical Considerations}

For analysis, Austria, Belgium, Switzerland, Denmark, Germany, France, Italy, Netherlands, Portugal, Spain, Sweden, Great Britain, Finland, Norway, and Ireland are considered as countries in Western Europe; Bulgaria, Czech Republic, Greece, Hungary, Lithuania, Poland, Ukraine, Turkey, Russia, Romania, and Slovakia as countries in Eastern Europe.

\section{Results}

Recruitment of patients for FIGARO-DKD began in September 2015 and was completed in October 2018. A total of 19,380 individuals, including 796 patients who were rescreened, were enrolled at 1,058 sites in 47 countries, resulting in 7,437 randomized patients. During the run-in or screening phase but before randomization, 1,552 patients switched over from FIDELIO-DKD. The screening failure rate in all patients enrolled was 61.6 and $18.1 \%$ in patients who switched. Eighty-three patients in FIGARO-DKD were excluded from the full analysis set due to substantial Good Clinical Practice violations, resulting in $7,354(98.9 \%)$ patients in whom the primary analysis will be performed.

More than $45 \%$ of patients were recruited in Europe, with slightly more patients being randomized in Eastern Europe compared to Western Europe, 15.1\% in North America, 22.1\% in Asia, and 3.7\% in Australia, New Zealand, and South Africa (region "Other").

Design and Baseline Characteristics of the FIGARO Trial
The overall trial population is predominately male $(69.4 \%)$ and white $(73.5 \%)$, with a mean age of 64.1 years and a mean duration of diabetes of 14.5 years. At baseline, mean glycosylated hemoglobin was $7.7 \%$, mean eGFR $67.8 \mathrm{~mL} / \mathrm{min} / 1.73 \mathrm{~m}^{2}$, mean serum potassium $4.3 \mathrm{mEq} / \mathrm{L}$, and median UACR $312 \mathrm{mg} / \mathrm{g}$. More than $60 \%$ of patients had an eGFR $\geq 60 \mathrm{~mL} / \mathrm{min} / 1.73 \mathrm{~m}^{2}$ and the proportion of patients with high or very high albuminuria at baseline was balanced.

At baseline, 4,198 patients (57.1\%) were taking ARBs and 3,130 (42.6\%) ACEIs, as requested by the protocol, and almost all patients (97.6\%) were on glucose-lowering medications. More than half of the patients $(54.1 \%)$ were using insulin, while metformin was the most frequently used glucose-lowering oral drug at baseline. The use of GLP-1 and SGLT2 inhibitors at baseline was low (7.5 and $8.3 \%$, respectively).

At baseline, nearly all patients (95.8\%) had arterial hypertension as concomitant disease and $30.8 \%$ diabetic retinopathy. Less than half (44.3\%) had CVD in the medical history: $30.9 \%$ had coronary artery disease, $17.0 \%$ myocardial infarction, $11.1 \%$ ischemic stroke, $15.9 \%$ peripheral artery disease, and $1.3 \%$ had a carotid endarterectomy. Only 7.6\% of all patients suffered from heart failure at baseline, which is related to the protocol-requested exclusion of all patients with HFrEF and New York Heart Association classes II-IV at run-in and screening. Further information about the baseline characteristics can be found in Table 2 and online supplementary Table 2.

\section{Discussion}

Treatment of CVD in T2D has evolved over the last few decades, resulting in a substantial decline in diabetesrelated CV complications [1]. On top of improvements in clinical care, the health system and promotion in general as well as behavioral changes, newer treatments such as GLP-1 agonists or SGLT2 inhibitors have also demonstrated a further reduction of CV events in people with T2D [10, 12, 38-41]. Despite this promising progress, T2D patients with CKD still exhibit residual cardiorenal morbidity and mortality.

The effects of canagliflozin on CV outcomes were investigated in the Canagliflozin CV Assessment Study, where a $14 \%$ risk reduction for death from CV causes nonfatal myocardial infarction, or nonfatal stroke was observed [37]. In the Canagliflozin and Renal Events in Diabetes with Established Nephropathy Clinical Evaluation study investigating canagliflozin in patients with 
Table 2. Baseline characteristics

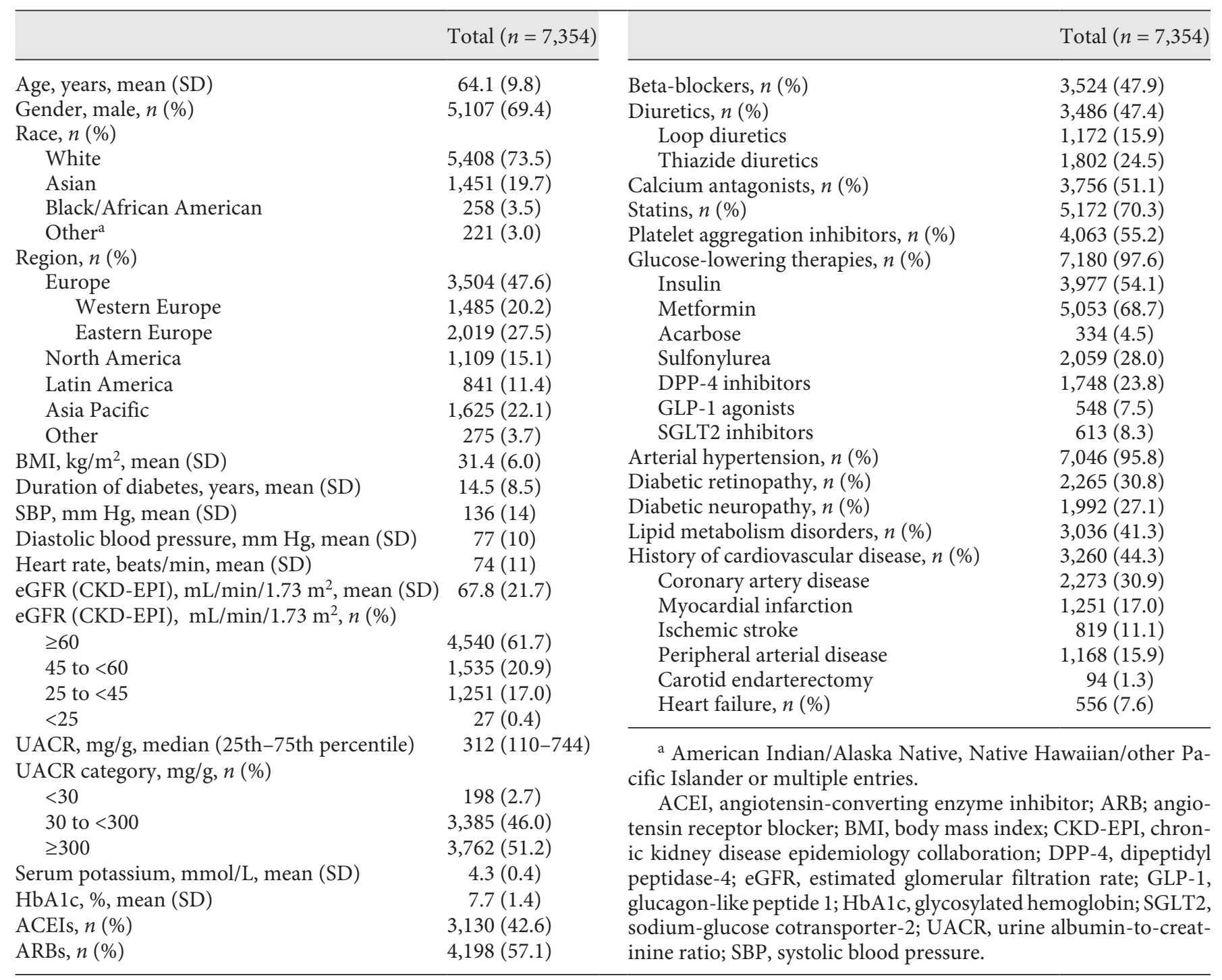

T2D and CKD with very high albuminuria, a 20\% risk reduction was observed for the same endpoint. In both studies, fewer effects of canagliflozin were seen in patients with an eGFR $\geq 60 \mathrm{~mL} / \mathrm{min} / 1.73 \mathrm{~m}^{2}$ or $\mathrm{UACR} \leq 1,000 \mathrm{mg} / \mathrm{g}$ at baseline $[39,42]$. In high-risk patients with an eGFR $<45 \mathrm{~mL} / \mathrm{min} / 1.73 \mathrm{~m}^{2}$ and UACR $>1,000 \mathrm{mg} / \mathrm{g}$ at baseline, the event rate per 1,000 patient-years was still high with 46.4 and 46.1 patients with an event despite treatment with canagliflozin, respectively. In a meta-analysis by Zelniker et al. [9], the effect of SGLT2 inhibitors on major adverse CV events (i.e., myocardial infarction, stroke, or $\mathrm{CV}$ death) was also less pronounced in patients with an eGFR $\geq 60 \mathrm{~mL} / \mathrm{min} / 1.73 \mathrm{~m}^{2}$ when compared to patients with an eGFR $<60 \mathrm{~mL} / \mathrm{min} / 1.73 \mathrm{~m}^{2}$.
Inflammation and fibrosis as well as oxidative stress mediated by MR overactivation have been well described in multiple organs and tissues, including the heart, vessels, and kidneys [43, 44], which contrasts to the renalselective SGLT2 expression in the early proximal tubule [45].

The currently available steroidal MRAs, spironolactone and eplerenone, have been investigated in clinical studies that varied between 4 and 52 weeks in duration and assessed the effects on albuminuria in different CKD populations. However, the effects of these MRAs on major $\mathrm{CV}$ outcomes in patients with CKD are unknown [46]. In a recent meta-analysis, Yang et al. [47] concluded that treatment with spironolactone in patients with mod- 
erate to severe (stage 3-4) CKD was not associated with a significant risk reduction in major adverse CV outcomes, hospitalization for heart failure, or all-cause or $\mathrm{CV}$ mortality compared to nonusers of spironolactone. They concluded, in addition, that the observed renoprotective benefits of spironolactone (relative risk reduction in ESRD by 34\%) may be offset by the hyperkalemia risk and recommended strategic treatments to prevent hyperkalemia.

Finerenone is a novel, nonsteroidal, selective MRA that is associated with less hyperkalemia compared to steroidal MRAs [48]. In the Antagonist Tolerability StudyDiabetic Nephropathy study, finerenone induced a dosedependent reduction of UACR, with the largest placebocorrected reduction $(-38 \%)$ in the group being treated with $20 \mathrm{mg}$ once daily. Finerenone dosages over 90 days of treatment resulted in small declines in eGFR $(\sim 2$ to $\left.4 \mathrm{~mL} / \mathrm{min} / 1.73 \mathrm{~m}^{2}\right)$ and blood pressure $(3-5 \mathrm{~mm} \mathrm{Hg})$ as well as small changes in serum potassium $(+0.2 \mathrm{mEq} / \mathrm{L})$ [36].

Due to its mode of action, beneficial cardiorenal effects - including CV risk reduction and delaying CKD progression - could result either as a direct consequence of the antifibrotic and anti-inflammatory efficacy of finerenone in cardiac and renal tissues or as an indirect consequence of improving cross-function of both end organs.

The combination of FIGARO-DKD and FIDELIODKD comprises the largest cardiorenal outcome program recruited to specifically investigate the occurrence of fatal and nonfatal CV events and progression of kidney disease in $>13,000$ CKD patients with T2D. Unlike previous outcome trials where the levels of albuminuria and kidney function were advanced into stages 3 and 4, FIGARO-DKD has also included a cohort of patients with lower levels of albuminuria (high albuminuria defined as UACR $\geq 30 \mathrm{mg} / \mathrm{g}$ but $<300 \mathrm{mg} / \mathrm{g}$ ) and stage 2 CKD (eGFR $60-90 \mathrm{~mL} / \mathrm{min} / 1.73 \mathrm{~m}^{2}$ ) but also patients with high levels of albuminuria (UACR $\geq 300 \mathrm{mg} / \mathrm{g}$ ) and stage 1 and 2 CKD (eGFR $\left.\geq 60 \mathrm{~mL} / \mathrm{min} / 1.73 \mathrm{~m}^{2}\right)$, a patient population at high risk of $\mathrm{CV}$ events. Complemented by the results of FIDELIO-DKD, the program will allow the evaluation of effects of finerenone in the broadest spectrum of CKD patients with T2D ever investigated.

The results of FIGARO-DKD will determine whether an optimally treated cohort of T2D patients with CKD at high risk of $\mathrm{CV}$ events and progression of their renal disease will experience cardiorenal benefits with the addition of finerenone to their treatment regimen. Results are expected in 2021.

Design and Baseline Characteristics of the FIGARO Trial

\section{Acknowledgments}

The authors express their sincere appreciation to all involved colleagues in the study centers for their participation in this trial. The authors would also like to acknowledge all the patients who are participating in this trial. The authors thank the study coordinators Michelle King, Laurence West, Angela May (all Bayer PLC, Reading, UK), Stefania Collamati (Bayer SpA, Milan, Italy), Lydia Christopher (SLS Services employee performing services for Bayer PLC, Reading, UK), Anna Lindroth (Bayer AB, Solna, Sweden), Cecilia Fedorov (PRA Health Sciences employee performing services for Bayer Inc., Mississauga, Canada), GoUne You (Bayer Korea Ltd., Seoul, South Korea), Dirk Alta (Bayer B.V., Mijdrecht, Netherlands), the data managers Jeannette Gerstner, Jacobus Buytendach (Bayer AG, Berlin, Germany), Martin Gregory (PRA Health Sciences employee performing services for Bayer AG, Berlin, Germany), and the Statistical Analysts Cosima Klein, Aziz Tuemer, and Dominik Habel (all Bayer AG, Berlin, Germany). Special thanks to Meike Brinker (Bayer AG, Wuppertal, Germany) for her continuous support of the program in different important roles.

\section{Statement of Ethics}

The study protocol was approved by international review boards, independent Ethics Committees, and competent authorities according to national and international regulations. FIGARODKD is conducted in accordance with the ICH Harmonised Tripartite Guideline for Good Clinical Practice. All participants provided written, informed consent before entering the study.

\section{Disclosure Statement}

L.M.R. has served as an advisor/speaker for AstraZeneca, Bayer, Daiichi-Sankyo, Medtronic, Novartis, and Recordati. R.A. is a member of data safety monitoring committees for AstraZeneca and Ironwood Pharmaceuticals; a member of steering committees of randomized trials for Akebia, Bayer, Janssen, GlaxoSmithKline, Relypsa, and Sanofi and Genzyme US Companies; a member of adjudication committees for Bayer, Boehringer Ingelheim, and Janssen; and a member of a scientific advisory board or a consultant for Bird Rock Bio, Celgene, Daiichi Sankyo, Inc., Eli Lilly, Relypsa, Reata, Takeda Pharmaceuticals, USA, and ZS Pharma; he has served as associate editor of the American Journal of Nephrology and Nephrology Dialysis and Transplantation and has been an author for UpToDate; and he has received research grants from the U.S. Veterans Administration and the National Institutes of Health. S.D.A. has received research support from Abbott Vascular and Vifor International, and personal fees from Boehringer Ingelheim, Bayer, AstraZeneca, Novartis, Vifor International, Impulse Dynamics, Respicardia, and St. Jude Medical. G.L.B. reports research funding, paid to the University of Chicago, from Bayer, Janssen, and Vascular Dynamics; he has acted as a consultant for Merck, Vascular Dynamics, Relypsa, Boehringer Ingelheim, Sanofi, Pfizer, Novo Nordisk, Ionis, and AstraZeneca; he is an editor of the American Journal of Nephrology, Nephrology and Hypertension, and section editor of UpToDate; and he is associate editor of 
Diabetes Care and Hypertension Research. G.F. reports that he is a committee member of trials and registries sponsored by Bayer, Novartis, Servier, Vifor, Medtronic, and Boehringer Ingelheim. C.N., P.K., A.J., and N.M. are full-time employees of Bayer AG, Division Pharmaceuticals, Germany. B.P. reports consultant fees for Bayer, AstraZeneca, Sanofi, scPharmaceuticals, SQ Innovation, G3 Pharmaceuticals, Sarfez, Vifor, Cereno, Ardelyx, KBP Biosciences and Windtree Pharmaceuticals; he has stock options for KBP Biosciences, SQ Innovation, Sarfez, scPharmaceuticals, Cereno and G3 Pharmaceuticals, and Relypsa; he also holds a patent for site-specific delivery of eplerenone to the myocardium (US patent \#9931412).

\section{Funding Sources}

FIGARO-DKD is a research study conducted and funded by Bayer AG.

\section{Author Contributions}

The sponsor designed the study in conjunction with the Executive Committee. L.M.R. wrote the first draft of the paper, had full access to the study design information, and had final responsibility for the decision to submit for publication. All authors provided input for the preparation of subsequent drafts and approved the final version for submission. Technical editorial assistance was provided by Lynda Chang, $\mathrm{PhD}$, of McCann Health, and was funded by Bayer AG. All authors reviewed and approved the manuscript.

\section{Appendix}

Program Management and Committees

FIGARO-DKD is a research study conducted and funded by Bayer AG. The study protocol was written by the sponsor in close collaboration with the members of the Executive Committee (EC).
The EC supervises the conduct of the program, supported the protocol development, and provides ongoing scientific guidance. The members of the EC are L.M.R., G.L.B., G.F., and B.P., as well as R.A. (Chairman of the Renal Clinical Event Committee) and S.D.A. (Chairman of the CV Clinical Event Committee). A steering committee including the EC members, worldwide country lead investigators, and additional content experts is responsible for scientific guidance and local implementation of the protocols. The authors are responsible for drafting and editing this design and baseline paper and its content.

\section{Data Monitoring Committee}

Periodic assessments of safety and efficacy are performed in FIGARO-DKD by an independent Data Monitoring Committee, which also oversees the safety and efficacy in FIDELIO-DKD. One interim analysis for overwhelming efficacy in terms of reducing the primary $\mathrm{CV}$ efficacy endpoint as well as the main secondary renal endpoint was prespecified to occur after accrual of $67 \%$ of the planned primary CV efficacy endpoints. Members of the independent Data Monitoring Committee are Murray Epstein (Chair), Aldo P. Maggioni (Chair), Glenn M. Chertow, Gerald DiBona, Jean Rouleau, Jose Lopez-Sendon, and Tim Friede (Statistician).

\section{Central Clinical Event Committee}

All potential outcome events are independently reviewed and adjudicated by a Clinical Event Committee, which consists of 3 subcommittees (cardiac, renal, and neuro). All potential outcome events are randomly distributed to 2 members of the appropriate subcommittee. If there is disagreement between the 2 initial reviewers, the event will be distributed to a third reviewer. If the third reviewer does not agree with either of the first 2 reviewers, the event will be reviewed at a panel meeting in order to reach a decision.

Members of the Renal Clinical Event Committee are R.A. (Chair), Pantelis Sarafidis, Alan Jardine, Phyllis August, Sankar Navaneethan, and Titte Srinivas; members of the Cardiac Clinical Event Committee are S.D.A. (Chair), Andrew Coats, Piotr Ponikowski, John Teerlink, Barry Greenberg, James Januzzi, Stephan von Haehling, and Wolfram Doehner; members of the Neuro Clinical Event Committee are Wolfram Doehner (Chair), Turgut Tatlisumak, Lauren Phillips, Carlos Kase, and Hans Diener.

\section{References}

1 Gregg EW, Li Y, Wang J, Burrows NR, Ali $\mathrm{MK}$, Rolka D, et al. Changes in diabetes-related complications in the United States, 19902010. N Engl J Med. 2014 Apr;370(16):151423.

2 Ogurtsova K, da Rocha Fernandes JD, Huang Y, Linnenkamp U, Guariguata L, Cho NH, et al. IDF Diabetes Atlas: global estimates for the prevalence of diabetes for 2015 and 2040. Diabetes Res Clin Pract. 2017 Jun;128:40-50.

3 Global Burden of Metabolic Risk Factors for Chronic Diseases Collaboration. Cardiovascular disease, chronic kidney disease, and diabetes mortality burden of cardiometabolic risk factors from 1980 to 2010: a comparative risk assessment. Lancet Diabetes Endocrinol. 2014 Aug;2(8):634-47.

4 Eriksen BO, Ingebretsen OC. The progression of chronic kidney disease: a 10-year population-based study of the effects of gender and age. Kidney Int. 2006 Jan;69(2):375-82.

5 Afkarian M, Sachs MC, Kestenbaum B, Hirsch IB, Tuttle KR, Himmelfarb J, et al. Kidney disease and increased mortality risk in type 2 diabetes. J Am Soc Nephrol. 2013 Feb; 24(2):302-8.

6 Wen CP, Chang CH, Tsai MK, Lee JH, Lu PJ, Tsai SP, et al. Diabetes with early kidney involvement may shorten life expectancy by 16 years. Kidney Int. 2017 Aug;92(2):38896.
7 Pálsson R, Patel UD. Cardiovascular complications of diabetic kidney disease. Adv Chronic Kidney Dis. 2014 May;21(3):27380.

8 American Diabetes Association. Living standards of medical care in diabetes. 2019. [accessed July 30, 2019]. Available from: https:// care.diabetesjournals.org/living-standards\# June.

9 Zelniker TA, Wiviott SD, Raz I, Im K, Goodrich EL, Bonaca MP, et al. SGLT2 inhibitors for primary and secondary prevention of cardiovascular and renal outcomes in type 2 diabetes: a systematic review and meta-analysis of cardiovascular outcome trials. Lancet. 2019 Jan;393(10166):31-9. 
10 Husain $M$, Birkenfeld AL, Donsmark M, Dungan K, Eliaschewitz FG, Franco DR, et al.; PIONEER 6 Investigators. Oral semaglutide and cardiovascular outcomes in patients with type 2 diabetes. N Engl J Med. 2019 Aug; 381(9):841-51.

11 Marso SP, Bain SC, Consoli A, Eliaschewitz FG, Jódar E, Leiter LA, et al.; SUSTAIN-6 Investigators. Semaglutide and cardiovascular outcomes in patients with type 2 diabetes. N Engl J Med. 2016 Nov;375(19):183444.

12 Marso SP, Daniels GH, Brown-Frandsen K, Kristensen P, Mann JF, Nauck MA, et al.; LEADER Steering Committee; LEADER Trial Investigators. Liraglutide and cardiovascular outcomes in type 2 diabetes. N Engl J Med. 2016 Jul;375(4):311-22.

13 Favre J, Gao J, Zhang AD, Remy-Jouet I, Ouvrard-Pascaud A, Dautreaux B, et al. Coronary endothelial dysfunction after cardiomyocyte-specific mineralocorticoid receptor overexpression. Am J Physiol Heart Circ Physiol. 2011 Jun;300(6):H2035-43.

14 Fraccarollo D, Galuppo P, Hildemann S, Christ M, Ertl G, Bauersachs J. Additive improvement of left ventricular remodeling and neurohormonal activation by aldosterone receptor blockade with eplerenone and ACE inhibition in rats with myocardial infarction. J Am Coll Cardiol. 2003 Nov;42(9): 1666-73.

15 Fraccarollo D, Galuppo P, Schraut S, Kneitz S, van Rooijen N, Ertl G, et al. Immediate mineralocorticoid receptor blockade improves myocardial infarct healing by modulation of the inflammatory response. Hypertension. 2008 Apr;51(4):905-14.

16 López-Andrés N, Martin-Fernandez B, Rossignol P, Zannad F, Lahera V, Fortuno MA, et al. A role for cardiotrophin-1 in myocardial remodeling induced by aldosterone. Am J Physiol Heart Circ Physiol. 2011 Dec;301(6): H2372-82.

17 Ponikowski P, Voors AA, Anker SD, Bueno H, Cleland JG, Coats AJ, et al.; ESC Scientific Document Group. 2016 ESC Guidelines for the diagnosis and treatment of acute and chronic heart failure: the Task Force for the diagnosis and treatment of acute and chronic heart failure of the European Society of Cardiology (ESC)Developed with the special contribution of the Heart Failure Association (HFA) of the ESC. Eur Heart J. 2016 Jul; 37(27):2129-200

18 Edner M, Benson L, Dahlström U, Lund LH. Association between renin-angiotensin system antagonist use and mortality in heart failure with severe renal insufficiency: a prospective propensity score-matched cohort study. Eur Heart J. 2015 Sep;36(34):2318-26.

19 Edwards NC, Steeds RP, Stewart PM, Ferro CJ, Townend JN. Effect of spironolactone on left ventricular mass and aortic stiffness in early-stage chronic kidney disease: a randomized controlled trial. J Am Coll Cardiol. 2009 Aug;54(6):505-12.
20 Eschalier R, McMurray JJ, Swedberg K, van Veldhuisen DJ, Krum H, Pocock SJ, et al.; EMPHASIS-HF Investigators. Safety and efficacy of eplerenone in patients at high risk for hyperkalemia and/or worsening renal function: analyses of the EMPHASIS-HF study subgroups (Eplerenone in Mild Patients Hospitalization And SurvIval Study in Heart Failure). J Am Coll Cardiol. 2013 Oct;62(17): 1585-93.

21 Matsumoto Y, Mori Y, Kageyama S, Arihara K, Sugiyama T, Ohmura H, et al. Spironolactone reduces cardiovascular and cerebrovascular morbidity and mortality in hemodialysis patients. J Am Coll Cardiol. 2014 Feb; 63(6):528-36

22 Vardeny O, Wu DH, Desai A, Rossignol P, Zannad F, Pitt B, et al.; RALES Investigators. Influence of baseline and worsening renal function on efficacy of spironolactone in patients With severe heart failure: insights from RALES (Randomized Aldactone Evaluation Study). J Am Coll Cardiol. 2012 Nov;60(20): 2082-9.

23 Epstein M. Reduction of cardiovascular risk in chronic kidney disease by mineralocorticoid receptor antagonism. Lancet Diabetes Endocrinol. 2015 Dec;3(12):993-1003.

24 Jeunemaitre X, Chatellier G, Kreft-Jais C, Charru A, DeVries C, Plouin PF, et al. Efficacy and tolerance of spironolactone in essential hypertension. Am J Cardiol. 1987 Oct; 60(10):820-5

25 Pitt B, Zannad F, Remme WJ, Cody R, Castaigne A, Perez A, et al.; Randomized Aldactone Evaluation Study Investigators. The effect of spironolactone on morbidity and mortality in patients with severe heart failure. N Engl J Med. 1999 Sep;341(10):70917.

26 Accord-UK Ltd. Eplerenone Summary of Product Characteristics. 2017. [accessed August 27, 2019]. Available from: https://www. medicines.org.uk/emc/product/1773/smpc.

27 Pitt B, Bakris GL. New potassium binders for the treatment of hyperkalemia: current data and opportunities for the future. Hypertension. 2015 Oct;66(4):731-8.

28 Trevisan M, de Deco P, Xu H, Evans M, Lindholm B, Bellocco R, et al. Incidence, predictors and clinical management of hyperkalaemia in new users of mineralocorticoid receptor antagonists. Eur J Heart Fail. 2018 Aug; 20(8):1217-26.

29 Dhaybi OA, Bakris G. Mineralocorticoid antagonists in chronic kidney disease. Curr Opin Nephrol Hypertens. 2017 Jan;26(1):505 .

30 Bärfacker L, Kuhl A, Hillisch A, Grosser R, Figueroa-Pérez S, Heckroth $\mathrm{H}$, et al. Discovery of BAY 94-8862: a nonsteroidal antagonist of the mineralocorticoid receptor for the treatment of cardiorenal diseases. ChemMedChem. 2012 Aug;7(8):1385-403.

31 Pitt B, Filippatos G, Gheorghiade M, Kober L, Krum H, Ponikowski P, et al. Rationale and design of ARTS: a randomized, double-blind study of BAY 94-8862 in patients with chronic heart failure and mild or moderate chronic kidney disease. Eur J Heart Fail. 2012 Jun; 14(6):668-75.

32 Kolkhof P, Borden SA. Molecular pharmacology of the mineralocorticoid receptor: prospects for novel therapeutics. Mol Cell Endocrinol. 2012 Mar;350(2):310-7.

33 Kolkhof P, Delbeck M, Kretschmer A, Steinke W, Hartmann E, Bärfacker L, et al. Finerenone, a novel selective nonsteroidal mineralocorticoid receptor antagonist protects from rat cardiorenal injury. J Cardiovasc Pharmacol. 2014 Jul;64(1):69-78.

34 Grune J, Benz V, Brix S, Salatzki J, Blumrich A, Höft B, et al. Steroidal and nonsteroidal mineralocorticoid receptor antagonists cause differential cardiac gene expression in pressure overload-induced cardiac hypertrophy. J Cardiovasc Pharmacol. 2016 May;67(5):40211.

35 Grune J, Beyhoff N, Smeir E, Chudek R, Blumrich A, Ban Z, et al. Selective mineralocorticoid receptor cofactor modulation as molecular basis for finerenone's antifibrotic activity. Hypertension. 2018 Apr;71(4):599608

36 Bakris GL, Agarwal R, Chan JC, Cooper ME, Gansevoort RT, Haller H, et al.; Mineralocorticoid Receptor Antagonist Tolerability StudyDiabetic Nephropathy (ARTS-DN) Study Group. Effect of finerenone on albuminuria in patients with diabetic nephropathy: a randomized clinical trial. JAMA. 2015 Sep;314(9):88494.

37 Kidney Disease: Improving Global Outcomes (KDIGO) CKD Work Group. KDIGO 2012 clinical practice guideline for the evaluation and management of chronic kidney disease. 2013. [accessed July 30, 2019]. Available from: https://kdigo.org/wp.content/uploads/ 2017/02/KDIGO_2012_CKD_GL.pdf.

38 Gerstein HC, Colhoun HM, Dagenais GR, Diaz R, Lakshmanan M, Pais P, et al.; REWIND Investigators. Dulaglutide and cardiovascular outcomes in type 2 diabetes (REWIND): a double-blind, randomised placebocontrolled trial. Lancet. 2019 Jul;394(10193): 121-30.

39 Neal B, Perkovic V, Mahaffey KW, de Zeeuw D, Fulcher G, Erondu N, et al.; CANVAS Program Collaborative Group. Canagliflozin and cardiovascular and renal events in type 2 diabetes. N Engl J Med. 2017 Aug;377(7):64457.

40 Perkovic V, Jardine MJ, Neal B, Bompoint S, Heerspink HJ, Charytan DM, et al.; CREDENCE Trial Investigators. Canagliflozin and renal outcomes in type 2 diabetes and nephropathy. N Engl J Med. 2019 Jun;380(24): 2295-306.

41 Zinman B, Wanner C, Lachin JM, Fitchett D, Bluhmki E, Hantel S, et al.; EMPA-REG OUTCOME Investigators. Empagliflozin, cardiovascular outcomes, and mortality in type 2 diabetes. N Engl J Med. 2015 Nov;373(22): 2117-28. 
42 Mahaffey KW, Neal B, Perkovic V, de Zeeuw D, Fulcher G, Erondu N, et al.; CANVAS Program Collaborative Group. Canagliflozin for primary and secondary prevention of cardiovascular events: results from the CANVAS Program (Canagliflozin Cardiovascular Assessment Study). Circulation. 2018 Jan;137(4): 323-34.

43 Jaisser F, Farman N. Emerging roles of the mineralocorticoid receptor in pathology: toward new paradigms in clinical pharmacology. Pharmacol Rev. 2016 Jan;68(1):4975 .
44 Kolkhof P, Jaisser F, Kim SY, Filippatos G, Nowack C, Pitt B. Steroidal and novel nonsteroidal mineralocorticoid receptor antagonists in heart failure and cardiorenal diseases: comparison at bench and bedside. Handb Exp Pharmacol. 2017;243:271-305.

45 Vallon V, Platt KA, Cunard R, Schroth J, Whaley J, Thomson SC, et al. SGLT2 mediates glucose reabsorption in the early proximal tubule. J Am Soc Nephrol. 2011 Jan;22(1):10412.

46 Bolignano D, Palmer SC, Navaneethan SD, Strippoli GF. Aldosterone antagonists for preventing the progression of chronic kidney disease. Cochrane Database Syst Rev. 2014 Apr; (4):CD007004.
47 Yang CT, Kor CT, Hsieh YP. Long-term effects of spironolactone on kidney function and hyperkalemia-associated hospitalization in patients with chronic kidney disease. J Clin Med. 2018 Nov;7(11):459.

48 Pitt B, Kober L, Ponikowski P, Gheorghiade M, Filippatos G, Krum H, et al. Safety and tolerability of the novel non-steroidal mineralocorticoid receptor antagonist BAY 94-8862 in patients with chronic heart failure and mild or moderate chronic kidney disease: a randomized, double-blind trial. Eur Heart J. 2013 Aug;34(31):2453-63. 